hep-th/9911194

SLAC-PUB-8303

UCLA/99/TEP/36

SWAT-99-243

November, 1999

\title{
Perturbative Relations between Gravity and Gauge Theory ${ }^{\star}$
}

\author{
Zvi Bern ${ }^{1}$ \\ Department of Physics \\ University of California at Los Angeles \\ Los Angeles, CA 90095-1547, USA \\ Lance Dixon ${ }^{2}$ and Maxim Perelstein ${ }^{2}$ \\ Stanford Linear Accelerator Center \\ Stanford University \\ Stanford, CA 94309, USA \\ David C. Dunbar ${ }^{3}$ \\ Department of Physics \\ University of Wales Swansea \\ Swansea, SA2 8PP, UK \\ Joel S. Rozowsky ${ }^{4}$ \\ Institute for Fundamental Theory \\ Department of Physics \\ University of Florida \\ Gainesville, FL 32611
}

\begin{abstract}
We review the relations that have been found between multi-loop scattering amplitudes in gauge theory and gravity, and their implications for ultraviolet divergences in supergravity.
\end{abstract}

Submitted to Classical and Quantum Gravity

\footnotetext{
${ }^{1}$ Research supported in part by the US Department of Energy under grant DE-FG03-91ER40662.

${ }^{2}$ Research supported by the US Department of Energy under grant DE-AC03-76SF00515.

${ }^{3}$ Research supported in part by the Leverhulme Foundation.

${ }^{4}$ Research supported by the US Department of Energy under grant DE-FG02-97ER41029.

* Presented by L.D. at Strings 99, July 19-25, 1999, Potsdam, Germany.
} 


\section{Introduction}

Although Yang-Mills theory and Einstein gravity are both based on local symmetries, and include long-range forces at the classical level, they are very different theories. For example, Yang-Mills theory is renormalizable, and due to asymptotic freedom, highly nontrivial in the infrared region; whereas gravity is nonrenormalizable, and its ultraviolet structure is of the greatest interest theoretically. There are also great differences between the perturbative Feynman rules for the two theories: Those for Yang-Mills theory contain only three- and four-point vertices, while those for gravity can have arbitrarily many external legs. Nevertheless, string theory suggests the heuristic relationship,

$$
\text { gravity } \sim \text { (gauge theory) } \times \text { (gauge theory) }
$$

following from the representation of string amplitudes as integrals over world-sheet variables complex integrals for closed strings (gravity) and real integrals for open strings (gauge theory) and the factorization of the closed-string integrand into two copies of the open-string integrand. This relationship was made precise by Kawai, Lewellen and Tye (KLT) [1] for tree-level scattering amplitudes. In this talk we describe the relations between multi-loop scattering amplitudes in gravity and gauge theory that were obtained in ref. [2], and discuss some of their implications.

Multi-loop scattering amplitudes are of considerable interest in both nonabelian gauge theory and gravity (and their supersymmetric extensions). On the gauge theory side, there are practical implications for more precise predictions of jet rates and other QCD phenomena observed in collider experiments. To date, no jet rate computations have been carried out beyond next-to-leading order in the strong coupling $\alpha_{s}$, even though in many cases, such as $e^{+} e^{-}$annihilation into three jets, experiment demands one higher order. Two-loop scattering amplitudes are required to calculate such next-to-next-to-leading order corrections.

On the gravity side, ultraviolet properties are of primary interest. Although gravity is nonrenormalizable by power counting, and the conventional wisdom is that only string theory could possibly render it finite to all orders, in point of fact no divergence has yet been established for any supersymmetric theory of gravity in four dimensions. Nonsupersymmetric theories of gravity with matter

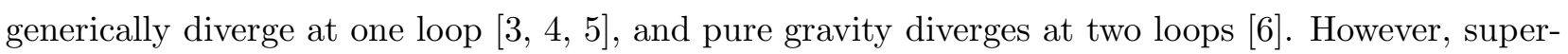
symmetry Ward identities (SWI) [7] forbid all possible one-loop [8] and two-loop [9] counterterms in any supergravity theory in $D=4$. Thus at least a three-loop calculation is required to definitively answer the question of the finiteness of four-dimensional supergravity. In addition, there is a candidate counterterm at three loops for all supergravities including the maximally extended version $(N=8)$ [10]. In ref. [2], divergences were computed in (higher-dimensional) $N=8$ supergravity, but only up to two loops. We expect, though, that the same techniques should be applicable beyond the two-loop level. In fact, our work suggests a natural conjecture for the divergences appearing at $L$ loops (see section (1). The conjecture would imply that $N=8$ supergravity in $D=4$ first diverges at five loops, not three loops.

Loop amplitudes in $D=11$ supergravity can also be used to extract information about $\mathrm{M}$ theory 11, 12]. Here the finite parts of the amplitudes are most important, particularly their dependence on the radii of one or two compactified dimensions. 
In principle one could investigate higher-loop relations between gravity and gauge theory in superstring theory using the world-sheet representation. However, at the multi-loop level this becomes technically rather difficult. Also, we would like to study the ultraviolet properties of particle theories of gravity. Recently, the KLT relations have been examined at the Lagrangian level, by introducing an auxiliary scalar field (i.e. the dilaton) into the Einstein-Hilbert action and carrying out appropriate field redefinitions 13. However, we won't pursue this direction further here. Instead we will use unitarity as a tool. Unitarity has proven very useful for one-loop QCD computations [14], and it has also been applied to two-loop $N=4$ super-Yang-Mills amplitudes [15]. The basic approach is to calculate the unitarity cuts of an amplitude, and then find functions that reproduce all such cuts. One can construct a perturbative bootstrap from tree amplitudes up to multi-loop amplitudes in this way. In the case of gravity, where the KLT relations express its tree amplitudes as roughly 'squares' of those of gauge theory, unitarity is particularly effective: The same algebraic steps employed in simplifying the gauge theory cuts can often be recycled in the (otherwise more complex) corresponding gravity cuts.

\section{Tree-level relations}

The starting point for investigating gravity-gauge theory relations via unitarity is the set of treelevel relations found by KLT [1]. The key observation is that any closed string vertex operator is a product of open string vertex operators,

$$
V^{\text {closed }}=V_{\text {left }}^{\text {open }} \times \bar{V}_{\text {right }}^{\text {open }} .
$$

This product structure is then reflected in the amplitudes, written as correlation functions of vertex operators.

For example, the open string tree amplitude for $n$ gluons is

$$
\left.A_{n} \sim \int \frac{d x_{1} \cdots d x_{n}}{\mathcal{V}_{a b c}} \prod_{1 \leq i<j \leq n}\left|x_{i}-x_{j}\right|^{k_{i} \cdot k_{j}} \exp \left[\sum_{i<j}\left(\frac{\epsilon_{i} \cdot \epsilon_{j}}{\left(x_{i}-x_{j}\right)^{2}}+\frac{k_{i} \cdot \epsilon_{j}-k_{j} \cdot \epsilon_{i}}{\left(x_{i}-x_{j}\right)}\right)\right]\right|_{\text {lin. }},
$$

where

$$
\mathcal{V}_{a b c}=\frac{d x_{a} d x_{b} d x_{c}}{\left|\left(x_{a}-x_{b}\right)\left(x_{b}-x_{c}\right)\left(x_{c}-x_{a}\right)\right|}
$$

and $x_{a}, x_{b}, x_{c}$ are any three of the $x_{i}$. In equation (3) we have suppressed the inverse string tension $\alpha^{\prime}$, and the 'lin.' denotes that after expanding the exponential one only keeps terms linear in each polarization vector $\epsilon_{i}$. The corresponding closed-string $n$-graviton amplitude is

$$
\begin{aligned}
M_{n} \sim \int \frac{d^{2} z_{1} \cdots d^{2} z_{n}}{\Delta_{a b c}} & \prod_{1 \leq i<j \leq n}\left(z_{i}-z_{j}\right)^{k_{i} \cdot k_{j}} \exp \left[\sum_{i<j}\left(\frac{\epsilon_{i} \cdot \epsilon_{j}}{\left(z_{i}-z_{j}\right)^{2}}+\frac{k_{i} \cdot \epsilon_{j}-k_{j} \cdot \epsilon_{i}}{\left(z_{i}-z_{j}\right)}\right)\right] \\
\times & \left.\prod_{1 \leq i<j \leq n}\left(\bar{z}_{i}-\bar{z}_{j}\right)^{k_{i} \cdot k_{j}} \exp \left[\sum_{i<j}\left(\frac{\bar{\epsilon}_{i} \cdot \bar{\epsilon}_{j}}{\left(\bar{z}_{i}-\bar{z}_{j}\right)^{2}}+\frac{k_{i} \cdot \bar{\epsilon}_{j}-k_{j} \cdot \bar{\epsilon}_{i}}{\left(\bar{z}_{i}-\bar{z}_{j}\right)}\right)\right]\right|_{\text {lin. }},
\end{aligned}
$$

where

$$
\Delta_{a b c}=\frac{d^{2} z_{a} d^{2} z_{b} d^{2} z_{c}}{\left|z_{a}-z_{b}\right|^{2}\left|z_{b}-z_{c}\right|^{2}\left|z_{c}-z_{a}\right|^{2}}
$$


and $z_{a}, z_{b}, z_{c}$ are any three of the $z_{i}$. In a helicity basis [16], one can write the graviton polarization tensor as a product of gluon polarization vectors, $\epsilon_{i}^{\mu \nu}( \pm)=\epsilon_{i}^{\mu}( \pm) \bar{\epsilon}_{i}^{\nu}( \pm)$.

The closed string integrand in (5) is a product of two open string integrands. This factorization holds for general closed-string states, not just gravitons, using the tensor product decomposition of closed string states in terms of open string ones, so that the vertex operator relation (2) can be applied.

From equations (3) and (5), various contour-integral deformations lead to relations between treelevel closed and open string amplitudes after all integrations have been performed [1]. Here we will need only the KLT relations in the limit $\alpha^{\prime} \rightarrow 0$ for the four- and five-point amplitudes [17]:

$$
\begin{aligned}
M_{4}^{\text {tree }}(1,2,3,4)= & -i s_{12} A_{4}^{\text {tree }}(1,2,3,4) A_{4}^{\text {tree }}(1,2,4,3), \\
M_{5}^{\text {tree }}(1,2,3,4,5)= & i s_{12} s_{34} A_{5}^{\text {tree }}(1,2,3,4,5) A_{5}^{\text {tree }}(2,1,4,3,5) \\
& \quad+i s_{13} s_{24} A_{5}^{\text {tree }}(1,3,2,4,5) A_{5}^{\text {tree }}(3,1,4,2,5),
\end{aligned}
$$

where the $M_{n}$ 's are the amplitudes in a gravity theory stripped of couplings, the $A_{n}$ 's are the colorordered subamplitudes in a gauge theory and $s_{i j} \equiv\left(k_{i}+k_{j}\right)^{2}$. We suppress all $\epsilon_{j}$ polarizations and $k_{j}$ momenta, but keep the ' $j$ ' labels to distinguish the external legs. The full amplitudes are given by

$$
\begin{aligned}
\mathcal{M}_{n}^{\text {tree }}(1,2, \ldots n) & =\left(\frac{\kappa}{2}\right)^{(n-2)} M_{n}^{\text {tree }}(1,2, \ldots n), \\
\mathcal{A}_{n}^{\text {tree }}(1,2, \ldots n) & =g^{(n-2)} \sum_{\sigma \in S_{n} / Z_{n}} \operatorname{Tr}\left(T^{a_{\sigma(1)}} T^{a_{\sigma(2)}} \cdots T^{a_{\sigma(n)}}\right) A_{n}^{\text {tree }}(\sigma(1), \sigma(2), \ldots, \sigma(n)),
\end{aligned}
$$

where $S_{n} / Z_{n}$ is the set of all permutations, but with cyclic rotations removed, $g$ is the gauge theory coupling constant, and $\kappa^{2}=32 \pi G_{N}$. The $T^{a_{i}}$ are fundamental representation matrices for the Yang-Mills gauge group $S U\left(N_{c}\right)$, normalized so that $\operatorname{Tr}\left(T^{a} T^{b}\right)=\delta^{a b}$.

Relations (7) and (8) can also be used when each external graviton state in $M_{n}$ is replaced by any of the 256 states of the $N=8$ supergravity multiplet. The Fock space decomposition

$$
\mid N=8 \text { SUGRA state }\rangle=\mid N=4 \text { super YM state }\rangle \otimes \mid N=4 \text { super YM state }\rangle
$$

should then be used to select the corresponding states for the two $A_{n}$ factors on the right-hand side of the relation.

\section{Loop amplitudes from unitarity}

Unitarity of the $S$ matrix, $S^{\dagger} S=1$, written in terms of the $T$ matrix defined by $S \equiv 1+i T$, reads

$$
2 \operatorname{Im} T_{i f}=\sum_{j} T_{i j}^{*} T_{j f}
$$

where $i$ and $f$ are initial and final states, and the 'sum' is over intermediate states $j$ (and includes an integral over intermediate on-shell momenta). Perturbative unitarity consists of expanding both 
sides of equation (11) in terms of coupling constants, $g$ for gauge theory and $\kappa$ for gravity, and collecting terms of the same order. For example, the imaginary (or absorptive) parts of one-loop four-point gauge amplitudes (order $g^{4}$ ) are given in terms of the product of two four-point tree amplitudes $\left(g^{2} \times g^{2}\right)$. The cuts in two-loop four-point gauge amplitudes (order $\left.g^{6}\right)$ are of two types: the product of a four-point tree amplitude and a four-point one-loop amplitude $\left(g^{2} \times g^{4}\right)$, and the product of two five-point tree amplitudes $\left(g^{3} \times g^{3}\right)$. In terms of the number of particles crossing the cut, the former is a two-particle cut, the latter a three-particle cut.

\subsection{Two-particle cutting equations}

The two-particle cuts in $N=4$ super-Yang-Mills theory have a very simple self-replicating structure [15]. The key equation is

$$
\sum_{S_{1}, S_{2} \in\{N=4\}} A_{4}^{\text {tree }}\left(-\ell_{1}^{S_{1}}, 1,2, \ell_{2}^{S_{2}}\right) \times A_{4}^{\text {tree }}\left(-\ell_{2}^{S_{2}}, 3,4, \ell_{1}^{S_{1}}\right)=-i \frac{s_{12} s_{23}}{\left(\ell_{1}-k_{1}\right)^{2}\left(\ell_{2}-k_{3}\right)^{2}} A_{4}^{\text {tree }}(1,2,3,4),
$$

where $\ell_{1,2}$ are the intermediate momenta, and $S_{1,2}$ label states of the $N=4$ multiplet. The $N=4$ labels corresponding to the external states with momenta $k_{i}$ have been suppressed, but equation (12) is valid for arbitrary combinations of external states. It is also valid for arbitrary (not just fourdimensional) momenta. One way to derive the equation is by working 'backwards' from the one-loop $N=4$ amplitudes first obtained using string theory [18].

Equation (12) can be represented graphically as

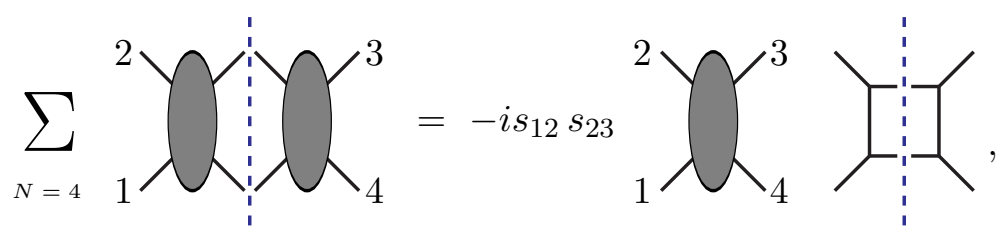

where the blobs represent tree amplitudes, and the two denominator factors in (12) are represented kinematically by the two internal propagators in the $\phi^{3}$ diagram on the far right. This representation makes clear that the one-loop two-particle cut is given by a cut scalar box integral, multiplied by the tree amplitude and a simple overall factor. The full one-loop amplitude is obtained simply by replacing the cut scalar box integral with the full scalar box integral ( $D$ is the spacetime dimension),

$$
\sum_{\infty} \equiv \frac{d^{D} \ell}{(2 \pi)^{D}} \frac{1}{\ell^{2}\left(\ell-k_{1}\right)^{2}\left(\ell-k_{1}-k_{2}\right)^{2}\left(\ell+k_{4}\right)^{2}}
$$

\section{2 $N=4$ iteration}

Because the dependence of the two-particle cut on the external $N=4$ states is just that of the tree amplitude, the two-particle cuts can be iterated easily. For example, the two-loop two-particle cut 
is given by

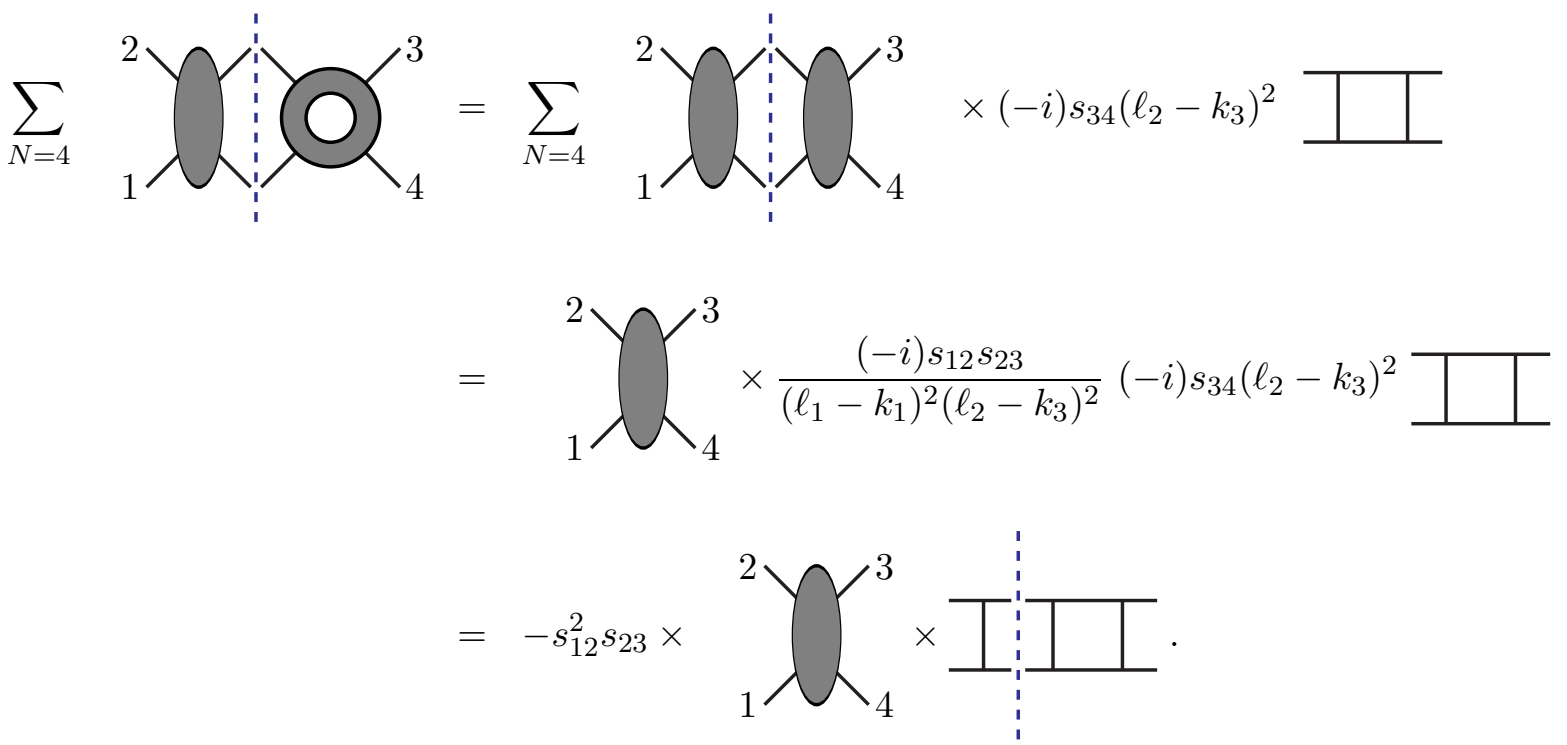

Clearly, the coefficients of all multi-loop ladder diagrams can be determined in this way; but so can the 'entirely two-particle constructible' diagrams, namely those which can be reduced to trees by successive two-particle cuts, for example

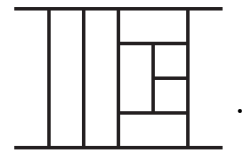

In general, each two-particle cut through a channel with momentum $\ell_{i}+\ell_{j}$ results in an additional factor of $\left(\ell_{i}+\ell_{j}\right)^{2}$ multiplying the $\phi^{3}$ integral [15].

At two loops, all terms in the $N=4$ super-Yang-Mills four-point amplitude are detectable by the iterated two-particle cuts. In order to confirm that these are the only terms, one must calculate the more complicated three-particle cuts [15]. Beginning at three loops (for nonplanar contributions), there are terms in the amplitude which do not have any two-particle cuts at all, so three-particle cuts are required just to guess their form.

The above discussion has been for color-ordered subamplitudes, which need to be dressed with appropriate color factors to produce the full gauge theory amplitude. For the entirely two-particle constructible terms, however, this dressing is very simple to describe: In a graphical representation of the color factors, where a Kronecker $\delta^{a b}$ is represented by an internal line and a structure constant $f^{a b c}$ by a three-vertex, one should multiply each kinematic $\left(\phi^{3}\right)$ graph by exactly the same colorfactor graph. 


\subsection{Recycling gauge theory into gravity}

The first step in repeating the above analysis for $N=8$ supergravity is to derive the corresponding two-particle cutting equation. Using the four-point KLT relation (7), followed by the Yang-Mills cutting equation (12), the appropriate product of two gravity amplitudes is

$$
\begin{aligned}
\sum_{S_{1}, S_{2} \in\{N=8\}} M_{4}^{\text {tree }}\left(-\ell_{1}^{S_{1}}, 1,2, \ell_{2}^{S_{2}}\right) \times M_{4}^{\text {tree }}\left(-\ell_{2}^{S_{2}}, 3,4, \ell_{1}^{S_{1}}\right) \\
=-s_{12}^{2}\left(\sum_{S_{1}, S_{2} \in\{N=4\}} A_{4}^{\text {tree }}\left(-\ell_{1}^{S_{1}}, 1,2, \ell_{2}^{S_{2}}\right) \times A_{4}^{\text {tree }}\left(-\ell_{2}^{S_{2}}, 3,4, \ell_{1}^{S_{1}}\right)\right) \\
\quad \times\left(\sum_{S_{1}, S_{2} \in\{N=4\}} A_{4}^{\text {tree }}\left(\ell_{2}^{S_{2}}, 1,2,-\ell_{1}^{S_{1}}\right) \times A_{4}^{\text {tree }}\left(\ell_{1}^{S_{1}}, 3,4,-\ell_{2}^{S_{2}}\right)\right) \\
=\left(s_{12} s_{23} A_{4}^{\text {tree }}(1,2,3,4)\right)^{2} \frac{s_{12}^{2}}{\left(\ell_{1}-k_{1}\right)^{2}\left(\ell_{2}-k_{3}\right)^{2}\left(\ell_{2}+k_{1}\right)^{2}\left(\ell_{1}+k_{3}\right)^{2}} \\
=\left(s_{12} s_{23} A_{4}^{\text {tree }}(1,2,3,4)\right)^{2}\left[\frac{1}{\left(\ell_{1}-k_{1}\right)^{2}}+\frac{1}{\left(\ell_{1}-k_{2}\right)^{2}}\right]\left[\frac{1}{\left(\ell_{2}-k_{3}\right)^{2}}+\frac{1}{\left(\ell_{2}-k_{4}\right)^{2}}\right] .
\end{aligned}
$$

In the last step we have performed a partial-fractioning of the denominators (using on-shell relations), in order to get a form which is recognizable as a sum of four different cut scalar box integrals, corresponding to $1 \leftrightarrow 2$ and $3 \leftrightarrow 4$ permutations of the integral in (14).

Equation (17) can be iterated whenever equation (12) can. Its structure implies that the coefficients of the corresponding $\phi^{3}$ integrals in $N=8$ supergravity are essentially the squares of those in $N=4$ super-Yang-Mills theory (once color factors have been removed from the latter) [2]. Again the two-particle cuts should be checked via three- (and higher-) particle cuts. The check for gravity at two loops is greatly simplified by using the five-point KLT relation (8). The resulting two-loop $N=8$ supergravity amplitude is given by

$$
\begin{aligned}
& \mathcal{M}_{4}^{2 \text {-loop }}(1,2,3,4)=-i\left(\frac{\kappa}{2}\right)^{6}\left[s_{12} s_{23} A_{4}^{\text {tree }}(1,2,3,4)\right]^{2}
\end{aligned}
$$

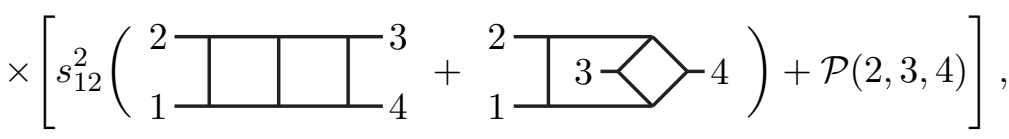

where ' $+\mathcal{P}(2,3,4)$ ' instructs one to add the five nontrivial permutations of legs $2,3,4$.

Beyond two loops, complete results are not yet available; a full analysis of higher-particle cuts must still be performed.

\section{Ultraviolet divergences in $N=8$ supergravity}

\subsection{Two loops}

Two-loop ultraviolet divergences in $N=8$ supergravity can be extracted directly from the two-loop scattering amplitude (18), by evaluating the divergences of the two $\phi^{3}$ double-box integrals that 
appear. Since each integral has 7 propagators and $2 D$ loop momenta in the integration measure, $\mathcal{M}_{4}^{2 \text {-loop }}(1,2,3,4)$ is manifestly finite for $D<7$. This behavior is better than what was predicted by power counting in an $N=4$ superspace formalism, which suggested that $N=8$ supergravity should diverge at two loops in $D=5$ and 6 19. The discrepancy is presumably due to the lack of a manifest $N=8$ invariance in the power-counting argument. In the direct cut-based calculation, the on-shell $N=8$ supersymmetric Ward identities are utilized in summing over the intermediate states crossing the cuts.

For $D \geq 7$, no cancellations occur, and there are divergences in every dimension of interest. The results are [2],

$$
\left.\mathcal{M}_{4}^{2 \text {-loop, } D=n-2 \epsilon}\right|_{\text {pole }}=\frac{C_{n}}{\epsilon(4 \pi)^{n}} \times\left(\frac{\kappa}{2}\right)^{6} \times s t u M_{4}^{\text {tree }}
$$

where

$$
\begin{aligned}
C_{7} & =\frac{1}{2} \frac{\pi}{3}\left(s^{2}+t^{2}+u^{2}\right) \\
C_{9} & =\frac{1}{4} \frac{-13 \pi}{9072}\left(s^{2}+t^{2}+u^{2}\right)^{2} \\
C_{10} & =\frac{1}{12} \frac{-13}{25920} \operatorname{stu}\left(s^{2}+t^{2}+u^{2}\right), \\
C_{11} & =\frac{1}{48} \frac{\pi}{5791500}\left(438\left(s^{6}+t^{6}+u^{6}\right)-53 s^{2} t^{2} u^{2}\right),
\end{aligned}
$$

and $s=s_{12}, t=s_{23}, u=s_{13}$. We omit the $D=8$ two-loop divergence because the $D=8$ theory diverges already at one loop, as can be seen easily by inspecting the box integral (14). New counterterms appear at two loops, though, as one would expect from a nonrenormalizable theory. (There are no corresponding one-loop divergences in $D=9$ or $D=11$, in dimensional regularization, because all invariants have even dimension. In $D=10$, there is a cancellation of one-loop divergences between the three different box integrals, because $s+t+u=0$.)

The presence of a factor of $s t u M_{4}^{\text {tree }}$ in equation (19) implies that in all the above cases, for four graviton external states, the linearized counterterms take the form of derivatives acting on the operator

$$
t_{8} t_{8} R^{4} \equiv t_{8}^{\mu_{1} \mu_{2} \cdots \mu_{8}} t_{8}^{\nu_{1} \nu_{2} \cdots \nu_{8}} R_{\mu_{1} \mu_{2} \nu_{1} \nu_{2}} R_{\mu_{3} \mu_{4} \nu_{3} \nu_{4}} R_{\mu_{5} \mu_{6} \nu_{5} \nu_{6}} R_{\mu_{7} \mu_{8} \nu_{7} \nu_{8}}
$$

where the tensor $t_{8}$ is defined in equation (9.A.18) of ref. [20], plus the appropriate $N=8$ completion. When the indices are restricted to four dimensions, $t_{8} t_{8} R^{4}$ becomes equal to the Bel-Robinson tensor 21]. We note that the operator (21) appears in the tree-level superstring effective action [22]. It also appears as the one-loop counterterm for $N=8$ supergravity in $D=8$. Finally, by calculating the same amplitude in compactified supergravity, it can be argued that it appears in the M theory effective action at one loop [1], and with the above set of derivatives at two loops [12].

The result (20) shows that even $D=11$ supergravity diverges. The manifest $D$-independence of the cutting algebra allowed us to extend the calculation to $D=11$, even though there is no corresponding $D=11$ super-Yang-Mills theory. Although we have established a divergence, we have not given a full description of the multiplet of counterterms, in particular how it depends on the three-form potential $A_{\mu \nu \rho}$. Further work on the structure of the $D=11$ counterterm has been carried out in ref. [23]. 


\subsection{Beyond two loops (a conjecture)}

Although we have not performed a full calculation beyond two loops in either $N=4$ super-YangMills theory or $N=8$ supergravity, the structure of the entirely two-particle constructible terms leads to a natural conjecture for where divergences appear at $L$ loops. In the Yang-Mills case, for each additional loop the maximum number of powers of loop momentum in the numerator increases by two, corresponding to the insertion of $\left(\ell_{i}+\ell_{j}\right)^{2}$ mentioned in section 3.2. Thus, for $L>1$ loops we expect that the most divergent integrals have $2 L-4$ powers of loop momenta in the numerator. There are also three additional scalar propagators per loop. The integrals scale as

$$
\int\left(d^{D} p\right)^{L} \frac{\left(p^{2}\right)^{(L-2)}}{\left(p^{2}\right)^{3 L+1}} .
$$

(The $L=1$ case is special and must be treated separately.) These integrals are finite for

$$
D<\frac{6}{L}+4, \quad(L>1)
$$

The result (23) differs from expectations based on $N=2$ superspace power-counting [24]. Specifically, for dimensions $D=5,6$ and 7 the amplitudes first diverge at $L=6,3$ and 2 loops. The corresponding superspace arguments indicate that the first divergence may occur at $L=4,3$ and 2 , respectively. Although (23) is still only a conjecture, it is consistent with three-loop contributions that are not detectable by the two-particle cuts, and for which we have evaluated the three-particle cuts. More generally, it is also consistent with a subset of the $n$-particle cuts where so-called 'maximally helicity-violating' tree amplitudes appear on each side of the cut [2]. We suspect that the difference with ref. [24] is due to a lack of full manifest supersymmetry in the power-counting argument.

For $N=8$ supergravity, from insertions of $\left[\left(\ell_{i}+\ell_{j}\right)^{2}\right]^{2}$ factors in the two-particle cuts, we expect the scaling of the most divergent integrals at $L$ loops to be

$$
\int\left(d^{D} p\right)^{L} \frac{\left(p^{2}\right)^{2(L-2)}}{\left(p^{2}\right)^{3 L+1}} .
$$

This leads to a conjectured finiteness condition,

$$
D<\frac{10}{L}+2, \quad(L>1) .
$$

In particular, we expect no three-loop divergence to appear in $D=4$ - contrary to expectations from an $N=4$ superspace analysis [19, 24]. We expect the first $R^{4}$-type counterterm to occur at five loops instead. The divergence should have the same kinematical structure as the $D=7$ divergence in (19), but with a different non-vanishing numerical coefficient.

Ref. [12] argues that the coefficients of operators with exactly four derivatives acting on $t_{8} t_{8} R^{4}$ in the $\mathrm{M}$ theory effective action are completely accounted for by the two-loop amplitudes. However, the scaling behavior in (24), inferred from the two-particle cuts, suggests nonvanishing contributions of this type from all $L>2$. A full three-loop supergravity calculation would be very welcome, to address both the expected finiteness in $D=4$, and whether there are $\partial^{4} t_{8} t_{8} R^{4}$ contributions. 


\section{Conclusions}

The heuristic relation (1) between gravity and gauge theory is a very useful way to think about gravity, at least perturbatively. Unitarity allows one to bootstrap the tree-level (KLT) versions of (1) up to the multi-loop level. Thus gravity calculations can be performed by recycling the simpler Yang-Mills calculations. Although we have discussed primarily the maximally supersymmetric case, where explicit calculations are the simplest, there should be applications to more general cases as well. Indeed, special one-loop helicity amplitudes with arbitrary numbers of external gravitons have been computed in this way, in both $N=8$ supergravity and pure gravity [25].

We have learned that supergravity amplitudes are less divergent than previously expected. In $D=4$, it is still true that no supergravity divergence has yet been firmly established. Indeed, our expectations in the maximally supersymmetric case, $N=8$, are for the first divergence to occur

only at five loops, not three loops. Further work in this direction, using the techniques described here, could help to remedy this situation.

\section{References}

[1] H. Kawai, D.C. Lewellen and S.H. Tye, Nucl. Phys. B269, 1 (1986).

[2] Z. Bern, L. Dixon, D.C. Dunbar, M. Perelstein and J.S. Rozowsky, Nucl. Phys. B530, 401 (1998) hep-th/9802162.

[3] G. 't Hooft and M. Veltman, Annales Poincaré Phys. Theor. A20, 69 (1974).

[4] G. 't Hooft, Nucl. Phys. B62, 444 (1973).

[5] S. Deser and P. van Nieuwenhuizen, Phys. Rev. D10, 401, 411 (1974);

S. Deser, H. Tsao and P. van Nieuwenhuizen, Phys. Rev. D10, 3337 (1974).

[6] M.H. Goroff and A. Sagnotti, Nucl. Phys. B266, 709 (1986);

A.E.M. van de Ven, Nucl. Phys. B378, 309 (1992).

[7] M.T. Grisaru, H.N. Pendleton and P. van Nieuwenhuizen, Phys. Rev. D15, 996 (1977);

M.T. Grisaru and H.N. Pendleton, Nucl. Phys. B124, 81 (1977);

S.J. Parke and T.R. Taylor, Phys. Lett. B157, 81 (1985).

[8] M.T. Grisaru, P. van Nieuwenhuizen and J.A. Vermaseren, Phys. Rev. Lett. 37, 1662 (1976).

[9] M.T. Grisaru, Phys. Lett. 66B, 75 (1977);

E. Tomboulis, Phys. Lett. 67B, 417 (1977).

[10] R.E. Kallosh, Phys. Lett. B99, 122 (1981).

[11] M.B. Green, M. Gutperle and P. Vanhove, Phys. Lett. B409, 177 (1997) hep-th/9706175;

J.G. Russo and A.A. Tseytlin, Nucl. Phys. B508, 245 (1997) hep-th/9707134. 
[12] M.B. Green, H. Kwon and P. Vanhove, hep-th/9910055;

M.B. Green, in Class. Quantum Grav. 17 (proceedings of Strings 99).

[13] Z. Bern and A.K. Grant, Phys. Lett. B457, 23 (1999) hep-th/9904026.

[14] Z. Bern, L. Dixon and D.A. Kosower, Ann. Rev. Nucl. Part. Sci. 46, 109 (1996) hep$\mathrm{ph} / 9602280$.

[15] Z. Bern, J.S. Rozowsky and B. Yan, Phys. Lett. B401, 273 (1997) hep-ph/9702424.

[16] P. De Causmaecker, R. Gastmans, W. Troost and T.T. Wu, Phys. Lett. 105B, 215 (1981), Nucl. Phys. B206, 53 (1982);

R. Kleiss and W.J. Stirling, Nucl. Phys. B262, 235 (1985);

J.F. Gunion and Z. Kunszt, Phys. Lett. B161, 333 (1985);

Z. Xu, D.-H. Zhang and L. Chang, Nucl. Phys. B291, 392 (1987).

[17] F.A. Berends, W.T. Giele and H. Kuijf, Phys. Lett. B211, 91 (1988).

[18] M.B. Green, J.H. Schwarz and L. Brink, Nucl. Phys. B198, 474 (1982).

[19] P.S. Howe, K.S. Stelle and P.K. Townsend, Nucl. Phys. B236, 125 (1984).

[20] M.B. Green, J.H. Schwarz, and E. Witten, Superstring Theory (Cambridge University Press, 1987).

[21] I. Robinson, unpublished;

L. Bel, Acad. Sci. Paris, Comptes Rend. 247, 1094 (1958), 248, 1297 (1959).

[22] D.J. Gross and E. Witten, Nucl. Phys. B277, 1 (1986).

[23] S. Deser and D. Seminara, Phys. Rev. Lett. 82, 2435 (1999) hep-th/9812136];

Class. Quant. Grav. 16, 2815 (1999) gr-qc/9905021.

[24] P.S. Howe and K.S. Stelle, Phys. Lett. B137, 175 (1984), Int. J. Mod. Phys. A4, 1871 (1989).

[25] Z. Bern, L. Dixon, M. Perelstein and J.S. Rozowsky, Nucl. Phys. B546, 423 (1999) hepth/9811140]. 